\title{
Scheme Design of Home Electricity Management System based on Smart Electricity Controller
}

\author{
Shi Su, a , Guipeng Zhang ${ }^{2, b}$, Yongzhong Zhang ${ }^{2, c}$, Zhengde Shi ${ }^{2, d}$, Bowen \\ Zheng ${ }^{2, \mathrm{e}}$, Xuechun $\mathrm{Li}^{2, \mathrm{f}}$ \\ ${ }^{1}$ Electric Power Research Institute Co., Ltd. of Yunnan Power Grid, Kunming 650217, China \\ ${ }^{2}$ Yuxi Power Bureau of Yunnan Power Co., Ltd, Yuxi 653199, China \\ apowertoyou1@163.com, ${ }^{\mathrm{b}}$ 609832658@qq.com, ${ }^{\mathrm{c}}$ 781673091@qq.com, ${ }^{\mathrm{d}}$ 121668686@qq.com, \\ ezhengbowen111@163.com, ${ }^{\mathrm{f}} \mathrm{xuechun1@126.com}$
}

\begin{abstract}
Keywords: smart controller; energy internet; internet of things; low voltage carrier communication technique; energy efficiency management; smart electricity consumption mode; active distribution network; Global Energy Internet
\end{abstract}

Abstract. On background of Energy Internet (EI) and active distribution network (ADN), a new type of smart controller based on low voltage carrier communication technique was developed, which could finish multi-interaction between the users and Power Supply Company (PSC) in aspects of energy flow and information flow, and the process was finished in distribution lines. Based on modern high precision measuring technique, the voltage and current in $220 \mathrm{~V} \mathrm{AC}$ lines are monitored in real time to obtain a large amount of electricity data of users, which provides sufficient data samples for smart electricity consumption mode analysis of home users, the demand side response modeling, and the work of ADN. The application research of designed smart controller on smart electricity consumption (SEC) was performed in four aspects, and it could support the "plug and play" of electrical equipment, based on the smart controller and other hardware devices and communication network, a smart home electricity management system was designed, and the smart electricity consumption mode was given and its mathematical model was built and the energy efficiency analysis and evaluation model were given. The designed smart home electricity management system could adequately take use of the home energy and photovoltaic generation, which have great significance to build Global Energy Internet in future.

\section{Introduction}

Since 1980s, the technique basis, organization and economic mode of energy industry have been gradually changing. The leading factors which promote the transformation include[1-3]: 1) The environment crisis, such as climatic change, due to the extensive use of fossil energy, has increasingly become rigorous; 2) With rapid development of many developing populous countries, the traditional industry and economy development mode of relying on non-renewable energy is difficult to continue; 3) The rapid development of renewable energy and information technology; 4) The ultimate goal of reform of the energy industry is to establish a more efficient, safe and sustainable energy utilization mode, which can solve the major and difficult problem of energy utilization faced by human society.

Jeremy Rifkin, a famous American scholar, firstly proposed the vision of the Energy Internet in his new book ${ }^{[4]}$ "The third industrial revolution", which caused widespread concern at home and abroad. He predicted that a new type of energy utilization system with the characteristics of deep combining with the new energy technology and information technology, namely, the "Energy Internet" would appear. The third industrial revolution, Energy Internet-centered, will produce a profound influence on the economic development mode and life style of human society. Jeremy Rifkin believed that the Energy Internet should have the following four characteristics ${ }^{[5-8]}: 1$ ) the renewable energy as the main primary energy source; 2) support the access of large scale distributed generation systems and distributed energy storage system; 3) realize wide area energy sharing based on Internet technology; 4) support the electrification of transportation system (i.e., the changing from the fuel vehicle to the electric vehicle). It can be seen from these characteristics that, the mainly connotations of Energy 
Internet advocated by Rifkin was taking use of Internet technique to achieve coordination of power supply, energy storage devices and load in a wide range; The ultimate goal is to realize the transformation from centralized fossil energy utilization to the usage of distributed renewable energy.

The number of distributed devices in the Energy Internet is huge, and if the problems of its access to system are both solved by power grid companies through artificial design and planning, which is obviously not realistic. The future Power Grid Corporations should be mainly responsible for the upgrading and expansion of power transmission and distribution system, and ensure that the energy management system of user side and the scheduling mechanism have adequate communication capabilities, and not specifically disturb the access of distributed devices ${ }^{[1,9]}$. This requires that the Energy Internet should have the ability to support plug and play of distributed devices, namely, develop the standard interfaces with ability of plug and play to support the distributed generation, energy storage, and controllable load and other devices. This standard interfaces include two aspects of physical and information. The physical interface should support the access of various distributed devices that meet the electrical standards; the information interface should support the identification and communication of a variety of distributed devices.

At present, some scholars have put forward the concept of energy router ${ }^{[6,10]}$, which realizes the function of voltage conversion between the middle and low voltage distribution network and the upper level power network, and the middle and low voltage power distribution network, as well as the function of the AC / DC conversion and information connection. The energy router achieves the interconnection between the regional sub-networks, and for each terminal user under the sub-network still needs to provide a standard interface, to achieve plug and play of each distributed device on user side ${ }^{[11,12]}$.

Based on this, this paper develops a new type of wall mounted intelligent controller based on carrier communication technique. The designed controller, combined with the low voltage carrier communication technology and based on the traditional sockets, which could realize the multiple interaction between the users and the power supply companies at in lines, at aspects of energy flow and information flow, thus support plug and play of electricity devices. Finally, based on the background of Energy Internet and ADN, the prospected of application of the smart controller in smart electricity consumption (SEC), especially for the non-productive load, is performed in four aspects, which is of great significance and guidance for the building of future smart home electricity management system and Network of Things system.

\section{Hardware Design of Smart Controller}

Design of Overall Hardware System. In order to solve the technical problems mentioned above, the smart controller designed in the thesis is based on low voltage carrier communication technology, which is composed of power conversion unit, signal measuring unit, switch unit, carrier communication unit and DSP data processing and control unit. Among them, the power conversion unit further includes common mode current suppression module, AC-DC voltage transformation module, and DC-DC voltage transformation module; the mentioned signal measuring unit further includes signal conditioning module and ADC conversion module; the signal conditioning module, further including the resistance capacitance voltage divider circuit, resistance detection circuit, and the differential amplifier circuit; The switch unit further comprises a relay switch circuit and optical coupler drive circuit; The DSP data processing and control unit further includes a DSP digital processor core circuit, the real-time clock circuit and data storage circuit.

The function of the smart controller designed in the paper can be concluded as follows. 1) on the basis of the traditional socket, realize the multiple information interaction between the users and the power supply companies in the energy flow and the information flow; 2) realize remote control for power equipment; realize real-time monitoring of voltage and current in $220 \mathrm{~V}$ AC lines, so that achieve a large number of electricity data of users, which can be uploaded to the Power Grid Corp's data service center through the carrier communication and the corresponding intelligent terminals, thus 
form the basic database of Energy Internet, and these data information can be used to provide basis for user's electricity utilization mode analysis, the demand side response modeling, and implementing of the ADN work, and so on. The system design principle of smart controller is shown in Fig. 1 as follows.

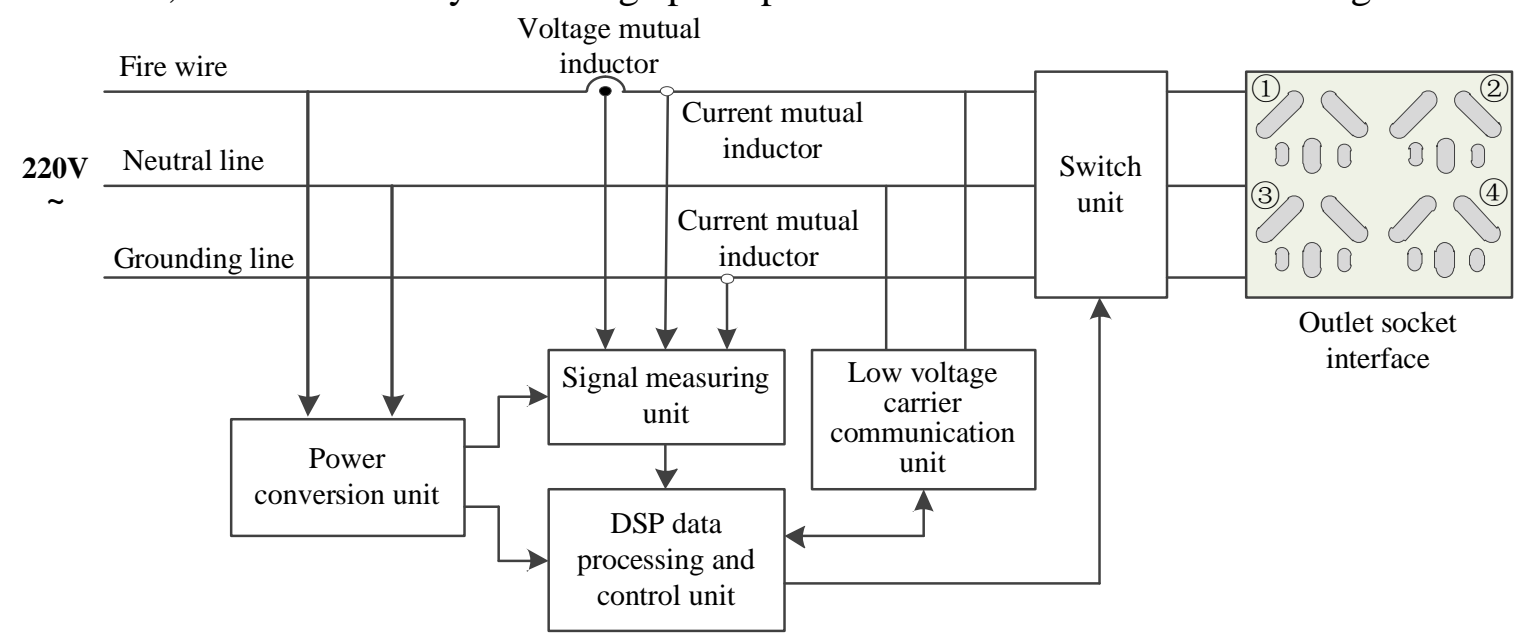

Fig. 1 Hardware structure design of smart controller

In Fig. 1, the input of the power conversion unit, the input of the signal measuring unit and the carrier communication unit are respectively connected in parallel with the $220 \mathrm{~V}$ lines; the switch unit is connected in series to the AC $220 \mathrm{~V}$ line; the output of the power conversion unit is respectively connected with the signal measuring unit, the DSP data processing and control unit, the carrier communication unit, and the switch unit; The DSP data processing and control unit is sequentially connected with the signal measuring module, the carrier communication unit, and the switching unit.

In the designed wall mounted smart controller, which is combined with the low voltage carrier communication technology, can realize multiple interaction between the users and the power supply companies in aspects of the energy flow and information flow; The switch module and the communication module are combined to realize the remote control of the power equipment; the modern high precision measurement technique which is adopted in the measuring module to perform real-time acquisition of voltage and current signal in $220 \mathrm{~V} \mathrm{AC}$ lines, thus obtain a large amount of electricity utilization data of users.

Design of Main Function Units. The internal detailed design structure of smart controller is shown in Fig. 2 below. In this diagram, the main functional units of smart controller include a power conversion unit, a signal measuring unit, a DSP data processing and control unit and a low voltage carrier communication unit. The main functional units are briefly summarized as follows.

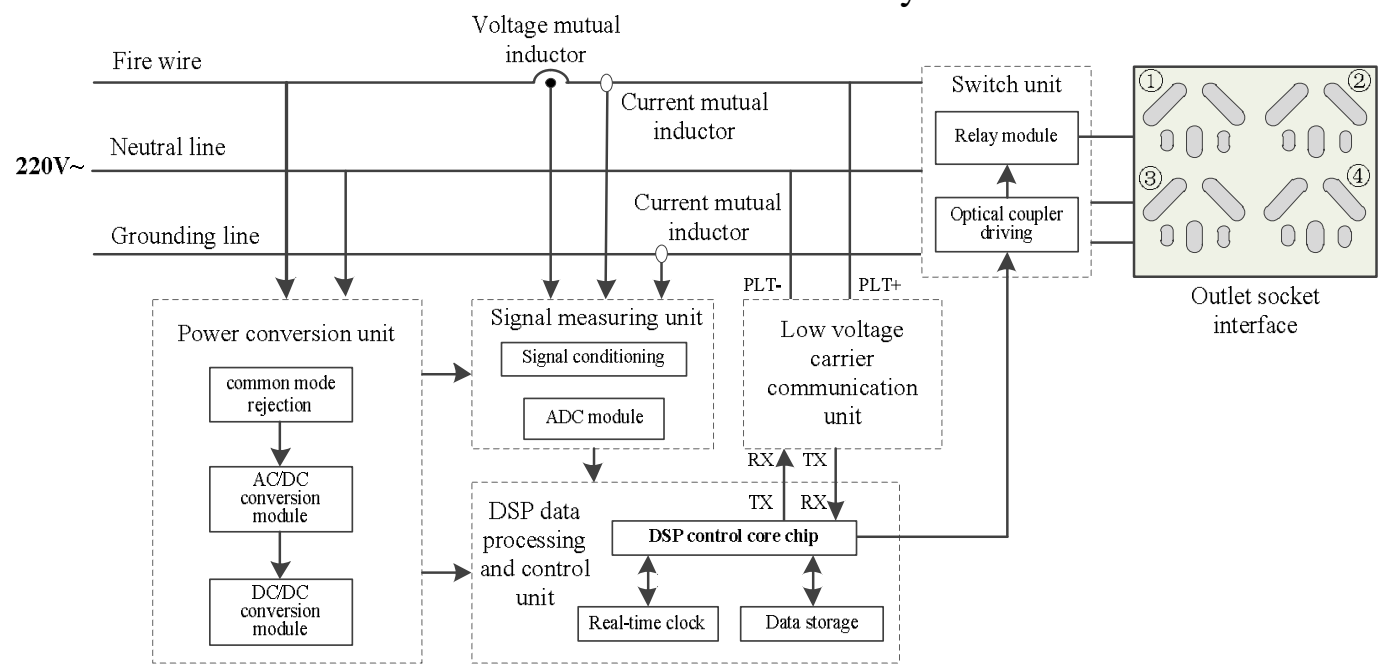

Fig. 2 Detailed design of internal structure in smart controlle

(1)The power conversion unit. It comprises a common mode signal suppression circuit, an AC-DC transformation circuit and a DC-DC transformation circuit, and they are connected in turn. Among 
them, the common mode signal suppression circuit can attenuate the carrier signal in the 220V AC line and prevent the power supply unit from interfering with the carrier communication unit; the AC-DC transformation circuit can convert the large AC voltage of $220 \mathrm{~V}$ AC lines to small DC voltage signal, so as to supply the other functional units of the smart controller; the DC-DC transformation circuit can convert the fixed amplitude of DC voltage output from the AC-DC conversion circuit to various amplitudes of DC voltage, so that meets the requirements of different functional units on power supply voltage.

(2) The signal measuring unit. It is mainly including signal conditioning circuit and ADC analog to digital conversion circuit ${ }^{[13]}$. Among them, the signal conditioning circuit is connected to the AC line, which can convert the large voltage signal in side of lines to a small voltage signal suitable for ADC acquisition; the ADC analog digital conversion circuit is connected between the signal conditioning circuit and the DSP data processing and control unit, and by which the analog signal can be converted into digital signal suitable for DSP processing.

(3)The DSP data processing and control unit. It is mainly composed of DSP core data processing chip, the real-time clock, and the data storage circuit ${ }^{[14]}$. Among them, the DSP digital signal processor is respectively connected with the ADC analog digital conversion circuit, the carrier communication unit, the switch unit, the real-time clock circuit and the data storage circuit, and which is core for the data processing and controlling; the real-time clock circuit can provide timing-based standard for measuring data; the data storage circuit can store the measuring data within a short time in case of power failure.

(4) The low voltage carrier communication unit. It mainly has the following characteristics: a) the time variability of communication channels; b) the frequency selectivity of communication channels; c) the noise interference is strong while the signal is attenuated rapidly.

Design of Low Voltage Carrier Communication Mode. The international newly-common narrowband power line carrier (PLC) standards include IEC 61334, the PRIME presented by the Spain Iberdrola power corporation, the G3-PLC initiated by the Electricite Reseau De France (ERDF), and the ITU G. 9955 /9956 announced by the International Telecommunication Uniform (ITU) and the IEEE1901.2 originated by the Institute of Electrical and Electronics Engineers (IEEE), et al ${ }^{[15,16]}$.

On the basis, the TCS081C chip can be adopted in the low voltage carrier communication unit, and the chip is independently developed by Qingdao Topscomm Communication CO., LTD. The block diagram of TCS081C chip is shown in Fig. 3 and the chip pin figure is shown in Fig. 4.

Base on the chip principle shown above, the TCS081C chip can achieve the data exchanging between the electronic terminal equipment based on power line communication network, and the data link layer adopts high-level data link control protocol (HDLC), and the application layer communication protocol is compatible with DL/T645-1997/2007 specification and it is optimized exclusively, and which can establish a reliable data connection by observing the DL/T645-1997/2007 protocol. The function of carrier communication of main station is achieved and meanwhile the system structure is simplified and the cost reduced and the stability of system improved. TCS081C chip performs the serial signal conversion between the power carrier signal and standard DL/T645-1997/2007 protocol of Topscomm protocol ${ }^{[17]}$.

In a summary, in order to select this kind of carrier communication chip of TCS081C, due to its excellent features as follows: 1) adopt spread spectrum communication technique; 2) software correlator and matched filter; 3) In differential $50 \mathrm{~Hz}$ AC power supply time period, can select the most conducive time communication for the transmission; 4) High performance digital signal processing technique; 5) BFSK modulation, half duplex communication; 6) Effective frame relay forwarding mechanism, support for relay depth of up to 16 levels; 7) Programmable network address, address filtering; 8) Strong system ability of communication; 9) Provide accurate information of node phase and channel characteristics; 10) 50bps, 100bps, 1200BPS, 600bps for each phase of carrier communication; 11) Serial communication rate of $9600 \mathrm{bps}, 14400 \mathrm{bps}, 19200 \mathrm{bps}, 28800 \mathrm{bps}$, 38400bps, 56000bps, 57600bps, 115200bps; 12) Hardware configuration of single and three phase 
operation mode; 13) Automatically log in power meter, and report the interrupt events; 14) Suitable temperature range (industry standard) is -40 degrees to +85 degrees.

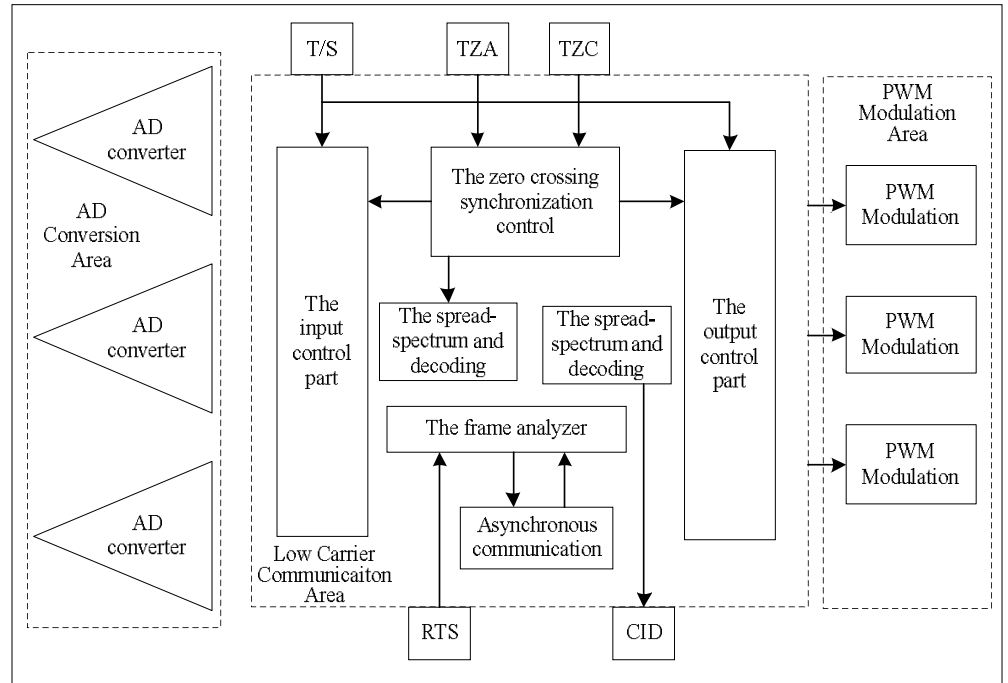

Fig. 3 The principle block diagram of TCS081C chip

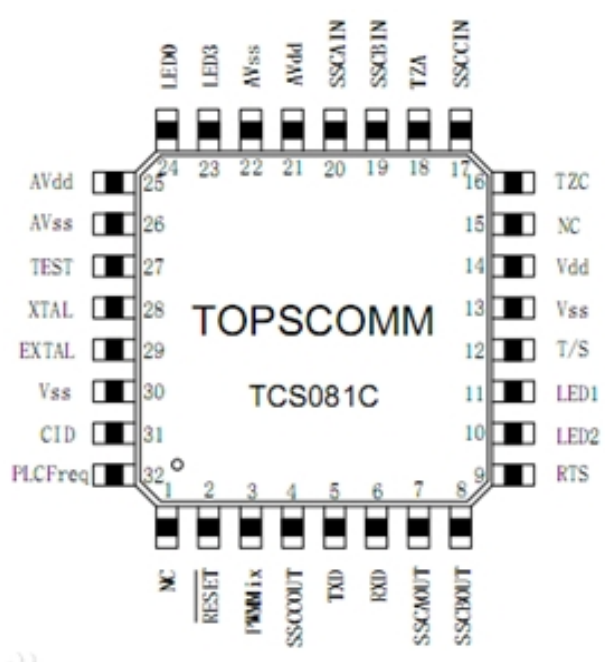

Fig. 4 The pins of TCS081C chip

The DSP processing module is inner set in the TCS081C chip, which can ensure the calculation requirement of carrier communication, and meanwhile, its pins of TZA, TZB, TZC are three-phase voltage zero crossing detection input, and the pins of SSCBIN, SSCCIN and SSCAIN are three-phase input of the carrier modulation signal, the pin of T/S is three-phase / single-phase carrier communication options, and the default is three-phase; the pin of LED0, LED1 and LED2 is respectively a channel indicating pulse, which indicates the $\mathrm{A}$ phase, $\mathrm{B}$ phase and $\mathrm{C}$ phase input of carrier modulation signal; the pin of LED3 is data receiving success indicator, and both high levels are effective; the pins of PLC_TX and PLC_RX are serial communication interfaces, and are communicated with series interface 4 of AT91SAM9260.

The internal zero crossing detection circuit provides a standard for the carrier communication transmission and receiving signal, and start working in zero voltage. According to the vector relationship of three-phase voltage, it's only need to detect two phases and the third phase can be detected by software algorithm; the zero crossing detection circuit completes the output of time synchronization signal TTL level then the AC current crosses zero, and the deviation of zero crossing of power is $\pm 4 \%$ and the error range of $50 \mathrm{~Hz} \mathrm{AC}$ current is 0 to $400 \mathrm{us}$, the error range of $\mathrm{AC}$ current of $60 \mathrm{~Hz}$ is 0 to $167 \mathrm{us}$. The carrier communication unit is connected to the power line through the carrier signal coupling circuit and the carrier signal to be received is coupled to the low voltage side, and the carrier power signal is transmitted to the power grid, and completes the impedance matching of signal.

\section{Contents of Main Scheme Design in Smart Home Electricity Management System based on Smart Electricity Consumption (SEC) Mode and Smart Controller}

There still has a very large increasing space for electricity consumption of national residents, on the basis, it's significant to take use of the smart controller designed in this thesis, and make study on load harmonics of residents, and further implement harmonic elimination, thus which has an important practical significance. Generally speaking, the low voltage loads of residents mainly consist of two aspects, i.e., one is the capacity filtering of diode bridge rectifier load, such as computer, color TV, etc.; the other is the voltage controlling load based on application of phase controlling principle, and this type of load controls the AC voltage and power by controlling the conduction angle of thyristors, such as single-phase induction voltage controller.

Due to the wide application of nonlinear equipment, so that the harmonic content in distribution network is very high, and which will cause the following serious situations, i.e., overload and overheating of distribution network transformer and the lines; mechanical vibrations of generator and 
motor; insulation damage of capacitor; voltage fluctuation and protection devices misoperation. Further, due to the superposition of three times zero sequence harmonics in the neutral line, it is easy to cause adverse consequences, such as the neutral line current is too large to burn out, and the electromagnetic force increases, and the neutral line voltage is too high. Therefore, taking full use of the real-time electricity consumption data monitored and stored by smart controller, and the uploading the data to the upper system software for electricity consumption analysis is significant in aspect of application. The upper software system is developed to study the non-productive users, such as home users, according to the real-time monitoring data, which can lay an important foundation for building smart home electricity management system and Network of Things system. In particular, the application prospects of smart controller in SEC are implemented in following four aspects.

SEC Mode and Efficiency Analysis and Its Optimization Study. Based on Energy Internet and ADN, the electricity consumption mode and its characteristics of non-productive users will be studied deeply, and make analysis of impacts of new energy generation, storage system and SEC on user utilization mode as well as its efficiency are done, and the concrete content is shown as follows.

1) Non-productive load classification and its utilization mode and feature analysis. Implement classification research on non-productive load, such as household electricity, building electricity (including living buildings, commercial buildings, office buildings, etc.), intelligent community, municipal electricity (including street lighting and other public services) and the urban functional districts, collect a large amount of electricity information, including the power quality evaluation index analysis, such as, the harmonic current analysis, shown in Fig.5 below, in which, the electricity consumption mode and its characteristic variables are summed up in multi-dimensional space including the time domain and frequency domain;

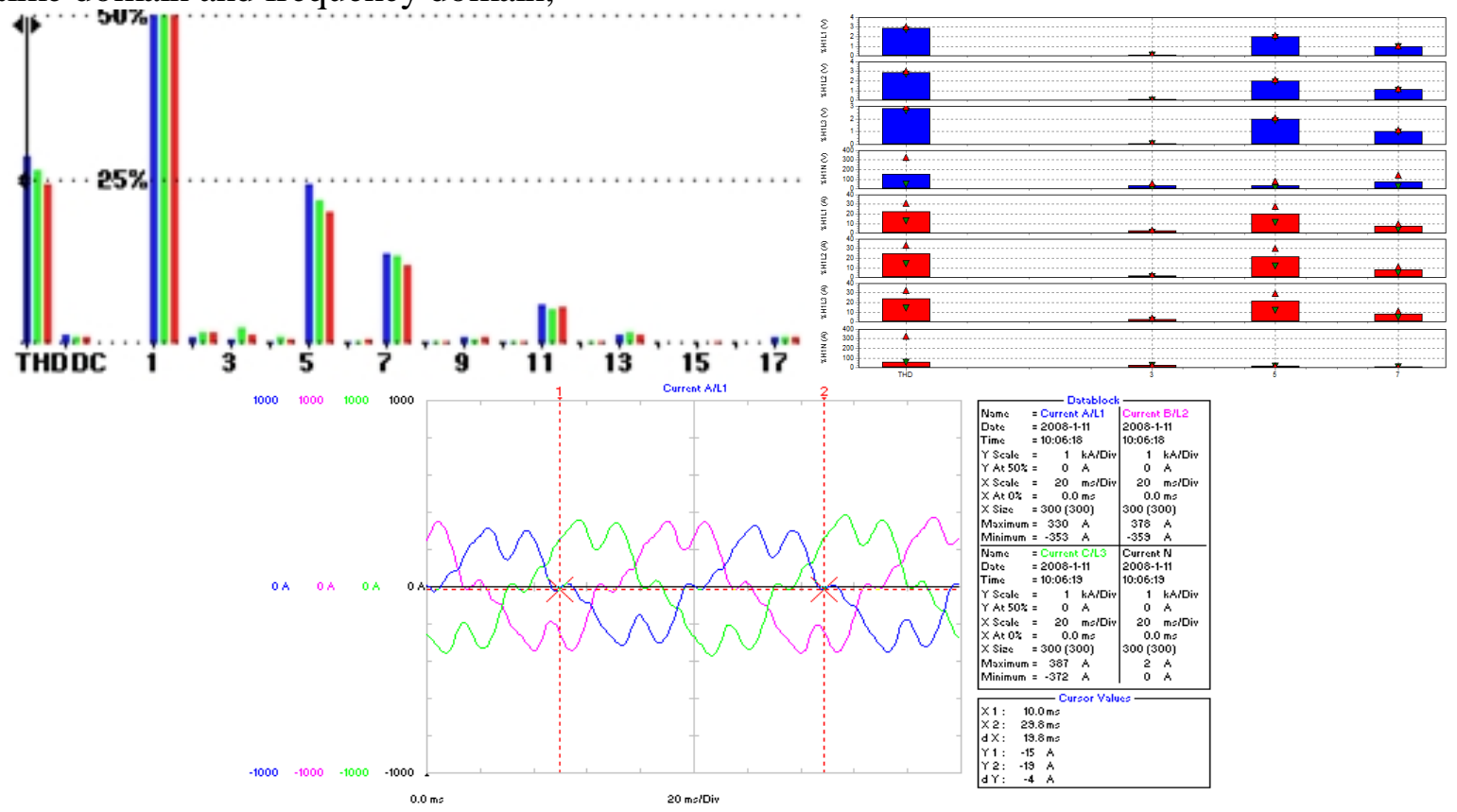

Fig. 5 The user harmonic analysis and wave diagram.

2) Study on the influence of new energy generation and energy storage on the electricity consumption mode. Implement research on the user's electricity consumption habits and plans which are changed by the new energy generation and energy storage system distributed in user load, as well as the impact of the size of distributed generation capacity on the regional electricity consumption mode, and modify its features and characteristic variables, so as to put forward the electricity consumption mode based on ADN.

3) Energy efficiency analysis method on user side and the efficiency analysis of each electricity consumption mode. Make analysis of user's typical electric equipment and its measuring mode, and mainly implement analysis on its electricity consumption and carbon emission, and derive the energy efficiency analysis method on user side and further make theoretical energy efficiency analysis and field 
verification in each consumption mode, and finally develop the energy efficiency analysis software which is faced with users, and which is used to perform energy efficiency analysis in different electricity consumption mode.

4) SEC mode analysis and the energy efficiency analysis study. In SEC mode, each consumption mode of electric equipment will change a lot. In case of many electricity consumption modes, make influence analysis of each mode on the conventional mode and especially combined with the comprehensive impact after access of new energy generation and energy storage, and implement efficiency analysis study in SEC mode, finally, aimed at the active load, put forward the new energy efficiency analysis methods.

5) Study the optimization of SEC mode. In the study, analyze the characteristics of different active loads, and make a unified optimization of user's new energy generation, energy storage and electricity consumption mode, and in case that guarantee stable operation of ADN, improve the energy efficiency level of users.

Unified Coordination and Control Study of SEC, New Energy Generation, and Energy Storage and Power Quality.1) Study the interaction influence mechanism of SEC, and new energy generation, energy storage and power quality. The SEC mode based on ADN refers to wide spread access of smart power system and new energy generation and storage devices and unified coordination and control, different systems access the power grid will interact with each other as well as lead to power quality problems in power grid inevitably. Therefore, the interaction between three of them and the influence mechanism of power quality due to its wide spread access grid should be studied, which forms a foundation for unified coordination and control of each system in SEC mode.

2) The study of power quality unified controller based on new energy generation and energy storage inverter system. The conventional new generation and energy storage devices access the inverter system of power grid, theoretically, which both have ability of reactive power outputting and harmonic controlling, while due to switch frequency and capacity limitation, the general inverter can only ensure the power factor close to 1, and have on ability of reactive power and harmonic controlling. Thus, it's necessary to develop new type of new energy generation and storage inverter controlling system, which can output active power normally meanwhile absorb the harmonic from other filter units, as well as adjust the reactive power output according to voltage requirements, thus avoid when the active power fluctuates in new energy generation system, resulting in distribution network voltage fluctuation and even transient rise and sag. The storage devices are set on the DC side, which can adjust the power fluctuation from new energy generation system, and set the photovoltaic power generation system as an example, the control strategy of power quality unified controller is shown in Fig. 6.

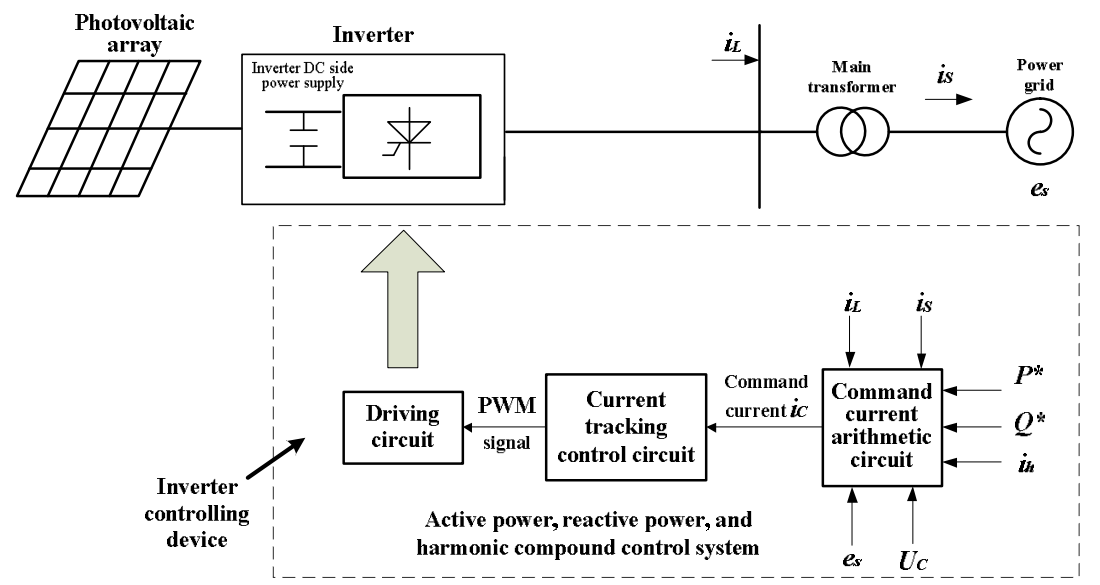

Fig. 6 The inverter control device schematic diagram contains the photovoltaic

3) The study of power quality unified coordination and control in case of SEC, new energy generation and storage system. The core value of ADN is active management, that is, in SEC mode, according to system operation situation, the load active power output, and system reactive power and voltage level are regulated and power grid harmonic is eliminated actively. In SEC mode, the electric 
equipment, the new energy generation and the storage system are both made to have ability of reactive power and harmonic output by changing the control strategy, and they can be responsible for demands of rapid reactive power compensation and harmonic elimination, and the compensation speed is fast, and can inhibit voltage problems of the voltage flicker and voltage sag caused by frequent fluctuation of active power. Finally, utilize the global optimization algorithm, the SEC equipment, and the new energy generation, and the storage system and grid power quality obtain a unified coordination and control, the concrete control strategy is shown in Fig. 7.

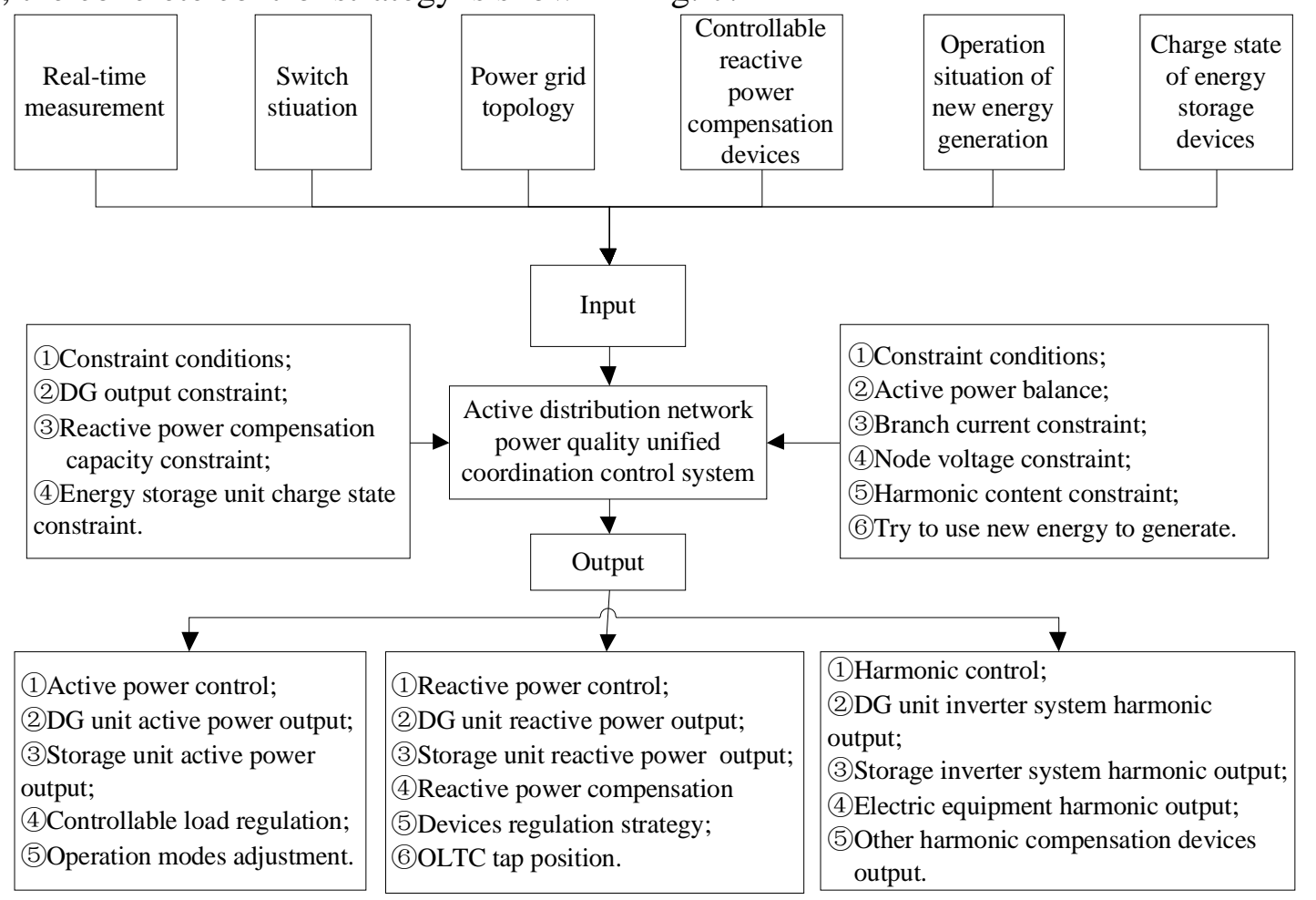

Fig. 7 The ADN power quality unified coordination control schematic diagram

The Study of SEC Mode Dynamic Optimization Technique based on Overall System.1) Impact modeling study of SEC on whole system. The SEC impacts the users, as well as the problems of the operation and construction, and efficiency level, and equipment utilization rate of power gird side and generation side, so that it's necessary to make analysis on impacts of electricity utilization deeply from aspect of whole system, and establish the mathematical model of electricity utilization mode impacts on whole system efficiency, and the equipment utilization rate, and the planning investment, etc., further, establish the multi-objective optimization mathematical model and in which multi-aspects of impacts, including the generation side, power grid side, and user side, as the optimal objection. It is shown in Figure 8 that perform control on each dispersed inversion controller and combine with demand side management of common users, thus we can further implement dynamic optimization study on the whole system electricity utilization mode.

2) The study of multi-objective SEC mode dynamic optimization based on whole system. It is performed to deeply analyze SEC mode optimization technique, according to the multi-objective Pareto-based and non-Pareto-based optimization methods (such as main objection method, linear weighted sum law, min-max method, etc.). Meanwhile, the multi-objections of mode dynamic optimization based on whole system include load curve optimization (peaking and valley-filling), operation and equipment investment, and renewable energy generation, etc. 


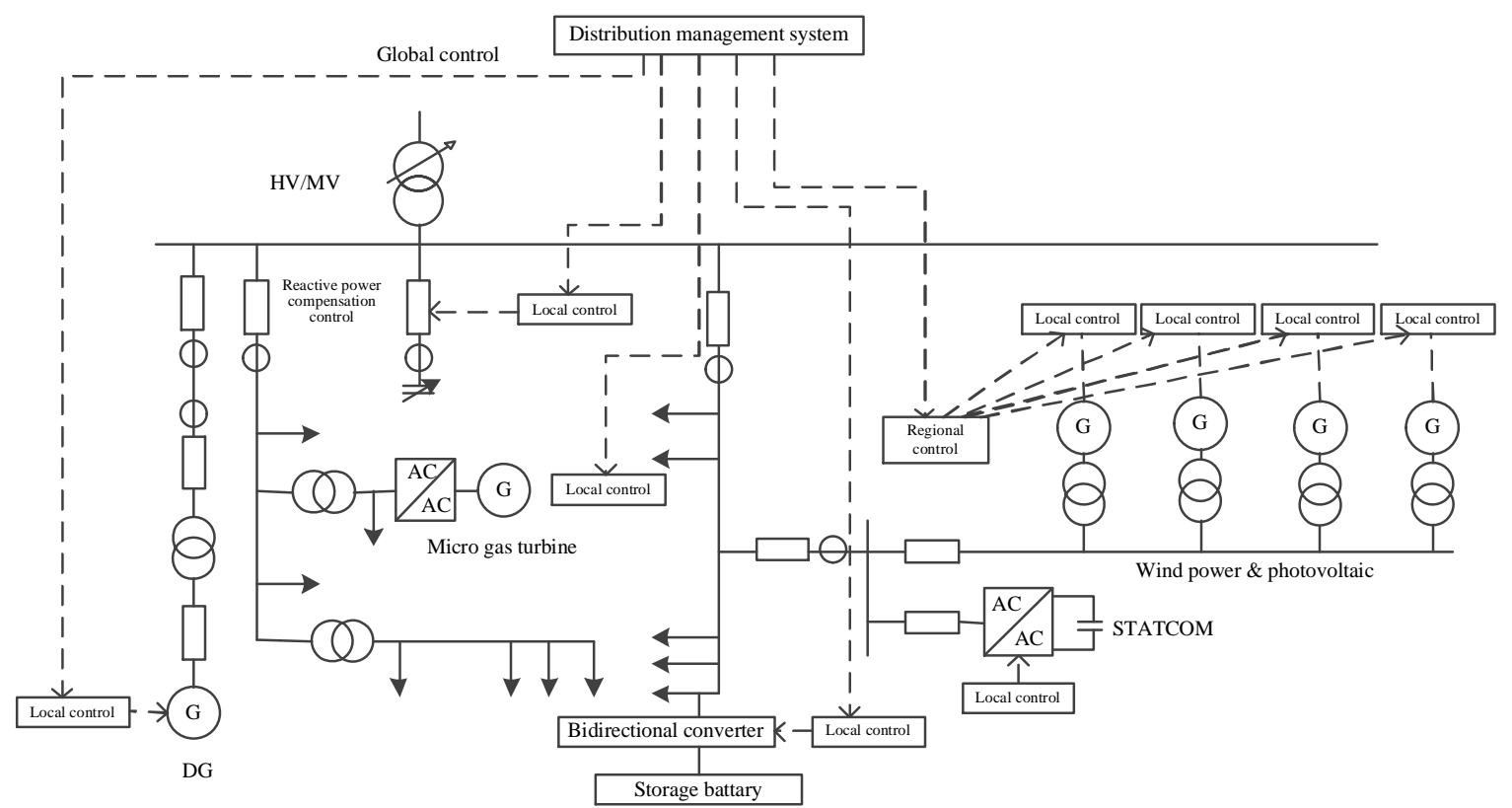

Fig.8 The whole system mode dynamic optimization framework schematic diagram

Development of SEC Management System and Demonstration Project Construction.1) Develop large numbers of small monitoring devices. Develop small devices with simple measurement and monitoring function (voltage and current and other real-time data), which just need small space, and are installed simply, and convenient for indoor installation.

2) Development of various electric equipment controller, that is the smart controller designed in this thesis, and the smart controller can control various electrical devices in real time and communicate with remote monitoring center in wireless. The remote control center update the control information (including electricity price, load situation, etc.) in real time, and the control commands are sent to the smart controllers, thus make the electrical equipment produce response, the concrete principle is shown in Fig. 9. The electricity utilization control methods are various, which include simple open-close control, as well as the most advanced real-time feedback control.

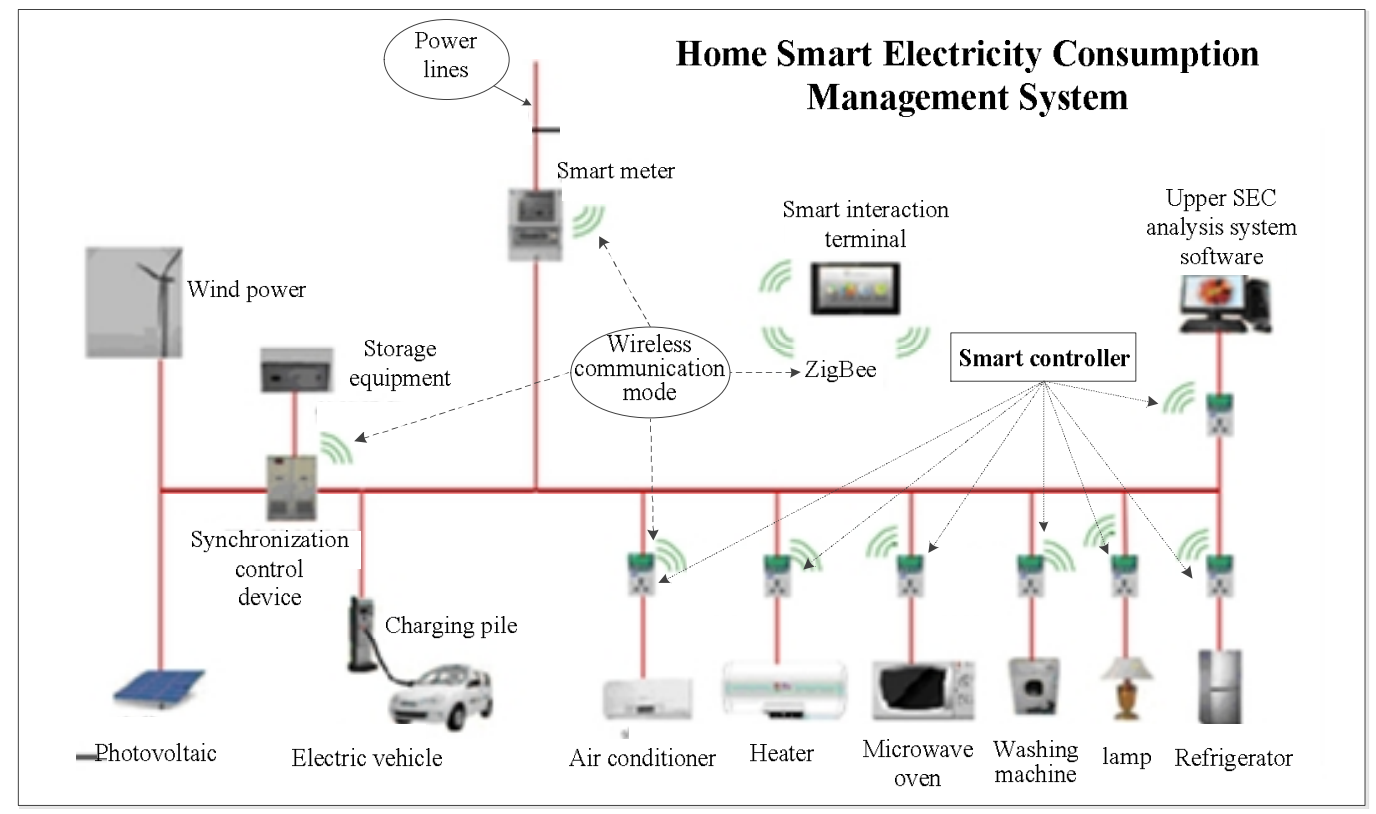

Fig.9 The user SEC management system structure schematic diagram

3) Development of SEC management system. Develop a whole system, which is a unified coordination optimization system, including the aforementioned new energy generation, energy storage, SEC, power quality, and consumption mode dynamic optimization, and can complete 
comprehensive functions of coordination control, advanced measurement, power quality control and efficiency management.

4) Design and implement of SEC management demonstration project. Based on the designed SEC management system mentioned above, establish a SEC management demonstration project, which can design or rebuild test building or district, and build SEC system. The designed SEC management system is performed a field verification, and further promote the research results of SEC management system actively, as well as explore the utilization mode of SEC management system for commercial operation and SEC service.

\section{Conclusions}

Based on Energy Internet and ADN background, aimed at the non-productive load, whose electricity consumption situation should be monitored and make analysis, then the consumption data can be uploaded to the upper software for electricity consumption mode dynamic analysis. On this basis, we can conclude that

1) a smart controller as the intelligent controller based on power line low voltage carrier technique and modularization was developed, which was designed as smart terminal interface to serve the Energy Internet, and achieved plug and play modes of various distributed electric equipment, so as to made information and energy bidirectional transmission between the distributed equipment and power grid. The smart controller provided plug and play unified interface for various distributed equipment, and was also compatible with a variety of common communication protocols, thus it was able to support intercommunication between various distributed equipment and power grid.

2) based on smart controller, prospects of its application in SEC studies were made in four aspects, i.e., the study of SEC mode and efficiency analysis and its optimization, the study of unified coordination and control of SEC, new energy generation, and energy storage and power quality, the study of SEC mode dynamic optimization technique based on whole system and the study of developing SEC management system and demonstration project construction. The approach to develop the upper software system for electricity consumption dynamic mode analysis could lay a significant foundation for building smart home electricity management system and Network of Things system and even the Energy Internet System (EIS).

3 ) the EIS has become research hotspot, it is believed that the EIS, which contains mobile internet, cloud computing, large data, Network of Things, energy system and modern manufacturing, will have a striding development in future, and the Third Industrial Revolution in which the Energy Internet as the core will bring profound influence on the economic development mode and life style of human society.

\section{Acknowledgements}

The authors gratefully acknowledge the support of the National Natural Science Foundation of China (No.51177051), and Science and Technology Projects of China Southern Power Grid (No. K-YN2014-145).

\section{References}

[1] C Y Dong, J H Zhao, F S Wen, Y S Xue, "From Smart Grid to Energy Internet: Basic Concept and Research Framework," Automation of Electric Power System, vol. 38, no. 15, pp. 1-11, 2015.

[2] H G Sun, Q L Guo, Z G Pan, "Energy internet: concept, architecture and frontier outlook", Automation of Electric Power System, vol. 39, no. 19, pp. 1-8, 2015.

[3] Y B Zha, T Zhang, Z Huang, Y Zhang, B L Liu, S J Huang, “Analysis of energy internet key technologies", The Journal of Science China: Information Sciences, vol. 57, no. 6, pp. 702-713, 2014. 
[4] RIFKIN J, "The third industrial revolution: how lateral power is transforming energy, the economy, and the world", New York: Palgrave MacMillan, 2011.

[5] J Y Wang, K Meng, J W Cao, Z H Cheng, L C Gao, C Lin, “Information technology for energy internet: a survey”, Journal of Computer Research and Development, vol. 52, no.5, pp. 1109-1126, 2015.

[6] J W Cao, K Meng, J Y Wang, M B Yang, Z Chen, W Z Li, C Lin, “An energy internet and energy routers”, Science China: Information Sciences, vol.44, no. 6, pp. 714-727, 2014.

[7] Y B Zha, T Zhang, S R Tan, Z Huang, W G Wang, "Understanding and thinking of the energy internet”, National Defense Science \& Technology, vol. 33, no. 5, pp. 1-6, 2012.

[8] J Y Wang, J H Guo, J W Cao, L C Gao, Z W Hu, J Zhou, Y Y Ming, Z W Fang, "Review on information and communication key technologies of energy internet", Smart Grid, vol. 3, no. 6, pp. $1-6,2015$.

[9] W Cai, H Zhao, J F Wang, C Lin, "A unifying network topological model of the energy internet macro-scope structure", Proceedings of the CSEE, vol. 35, no. 14, 2015.

[10] S M Tian, W P Luan, D X Zhang, C H Liang, Y J Sun, “Technical forms and key technologies on energy internet", Proceedings of the CSEE, vol. 35, no. 14, 2015.

[11] S Ci, "Energy information and internet-based management and its applications in distributed energy storage system", Proceedings of the CSEE, vol. 35, no. 14, 2015.

[12] T J Pu, K W Liu, N S Chen, X J Ge, J C Yu, D Wang, W Wang, "Design of ADN based urban energy internet architecture and its technological issues", Proceedings of the CSEE, vol. 35, no. 14, 2015.

[13] L F Cheng, J F Peng, Y X Li, X Z Hu, T Yu, "Development of special users' energy saving potential intelligent diagnosis detector based on DSP”, Electrical Measurement \& Instrumentation, vol. 51, no. 18, pp. 92-97, 2014.

[14] L F Cheng, B Zhou, T Yu, "Design and implementation of energy-saving potential automatically detecting online and rapid energy audit intelligent system”, Power System Protection and Control, vol. 42, no. 14, pp. 105-111, 2014.

[15] J J Qi, X P Chen, X S Liu, "Advances of research on low-voltage power line carrier communication technology”, Power System Technology, vol. 34, no. 5, pp. 162-172, 2010.

[16] F Chen, W G Zheng, C J Shen, P Zhou, W B Wu, "Low-voltage power line carrier communication technology and its application", Power System Protection and Control, vol. 37, no. 22, pp. 188-195, 2009.

[17] Y Zhang, D F Ren, A L Zhang, D Y Huang, "A solution for low-voltage carrier communication system based on Zigbee wireless technology", Power System Protection and Control, vol. 38, no. 10, pp. 110-113, 2010. 